\title{
La formazione in psicoterapia: Un dipinto in chiaroscuro
}

\author{
Marco Bani, ${ }^{1}$ Maria Grazia Strepparava, ${ }^{1}$ Giorgio Rezzonico ${ }^{1}$
}

Sommario: La formazione in psicoterapia rappresenta oggi per molti psicologi clinici una tappa importante e quasi obbligata per completare la propria professionalità; a fronte di questa richiesta crescente sono nate numerose scuole di formazione private, riconosciute dal Ministero dell'università, che si affiancano alle tradizionali scuole pubbliche. Per orientarsi in un panorama così complesso, uno strumento di accesso alle informazioni delle scuole dei diversi orientamenti è dato dai siti web di ciascuna di esse, cui si affiancano richieste telefoniche, e-mail e il colloquio di ammissione fatto dalla scuola. La domanda che guida il presente lavoro è se gli studenti-futuri utenti abbiano gli strumenti adeguati per fare una scelta consapevole, anche in ragione del grosso impegno di tempo e denaro che questa richiede.

Il lavoro ha analizzato i siti web di 201 sedi principali delle scuole di specializzazione riconosciute dal MIUR, valutando la presenza di alcuni dati relativi alle caratteristiche della scuola. I siti risultano sufficientemente aggiornati; tuttavia, alcune informazioni critiche (costi, analisi personale, supervisione) sono poco chiare, rendendo spesso problematica la scelta da parte degli studenti.

Parole chiave: formazione; psicoterapia; qualità; scuole; siti internet.

\footnotetext{
1 Multimedia Health Communication Laboratory, Facoltà di Medicina e Chirurgia, Università di Milano-Bicocca

Corrispondenza: marco bani@hotmail.com - Via Boccaccio 211 - 24059, Urgnano (BG)
}

Ricevuto: 22 Febbraio 2010 - Revisionato: 16 Maggio 2010 - Accettato: 20 Maggio: 2010 
Psychotherapy training is a lot like the weather. Everybody talks about it, but nobody does much about it. The aim of my talk is that we all do something about it.

(J. Norcross, 1996)

\section{Introduzione}

Il tema della formazione riveste un ruolo centrale nell'ambito della ricerca in psicoterapia, perché da un lato caratterizza la competenza di partenza degli psicoterapeuti, il punto di ancoraggio comune, che verrà poi affinato nel corso della pratica professionale secondo direzioni differenti (Orlinsky \& Rønnestad, 2005), e dall'altro rappresenta, pur tra numerose peculiarità, il file rouge che unisce gli psicoterapeuti a tutte le latitudini intorno ad alcuni nuclei chiave come quelli di relazione, transfert, empatia etc.

Tuttavia il tema della formazione in psicoterapia si caratterizza per una quantità di differenze che rendono difficile non solo farne un quadro comune, ma anche solo pensare che dietro l'etichetta di psicoterapeuta ci siano delle uniformità. Tali differenze riguardano essenzialmente le differenti metodologie didattiche delle specializzazioni, che a livello europeo e internazionale sono ancora più marcate, coinvolgendo la definizione stessa di psicoterapia, il ruolo riconosciuto alla pratica terapeutica e la relazione con altre professioni affini (counselor, social workers, etc.) (Botermans, 1996). Infatti, mentre in paesi come l'Italia, l'Austria (Psychotherapiegesetz, BGBl. Nr. 361/1990), la Germania (Psychotherapeutengesetz, 1998), la professione psicoterapeutica è regolamentata a livello ministeriale ed è prevista una definizione di competenze nonché una regolamentazione del percorso formativo, in altri paesi, come la Francia e l'Inghilterra, la regolamentazione è affidata alle società scientifiche o di categoria, pur in assenza di una definizione di psicoterapia e di un percorso formativo comune; infine la gran parte dei paesi dell'est europeo presenta al momento l'assoluta mancanza di regolamentazione, demandata in tutto e per tutto alle singole società scientifiche (Orlinsky, 2008).

Spostando l'attenzione a livello nazionale, la situazione risulta di gran lunga più regolamentata; infatti, con la legge del 1989 sull'ordinamento della professione di psicologo, sono stati definiti i criteri per l'esercizio della psicoterapia e per la formazione e successivamente con il decreto 
ministeriale $\mathrm{n}^{\circ} 509$ del $1989^{1}$ sono stati definiti in dettaglio gli standard di formazione, successivamente modificati in misura minima. Vediamo quindi quali sono i confini entro cui si muove la formazione.

Per l'istanza di riconoscimento gli istituti devono innanzitutto

"presentare l'ordinamento, la documentazione relativa alla validità del proprio indirizzo metodologico e teorico-culturale ed evidenze scientifiche che dimostrino la sua efficacia, la documentazione relativa all'esistenza, per i tirocini, di convenzioni con strutture o servizi pubblici e privati accreditati $[\ldots]$ la disponibilità di qualificato personale docente e non docente e di idonee strutture e attrezzature, necessarie all'efficace svolgimento dei corsi" (art. 2, D.M. 509/98).

Per quanto riguarda più specificamente la parte didattica i corsi devono avere durata almeno quadriennale, e con un monte ore di almeno 500 ore annuali di cui almeno 100 dedicate ad attività pratiche (cioè il tirocinio nelle strutture esterne alla scuola); le attività teoriche devono comprendere due sezioni: la prima "un'ampia parte di psicologia generale, di psicologia dello sviluppo, di psicopatologia e diagnostica clinica nonché la presentazione e discussione critica dei principali indirizzi psicoterapeutici"; la seconda "l'approfondimento specifico dell'indirizzo metodologico e teorico-culturale seguito dall'istituto" (art. 8, D.M. 509/98).

Relativamente alle attività pratiche viene evidenziata la necessità di

"una formazione coerente al tipo di indirizzo psicoterapeutico adottato dall'istituto; formazione che prevederà, oltre a specifici momenti formativi, la supervisione delle psicoterapie attuate dagli allievi durante l'addestramento pratico", e "per il tirocinio...documentate esperienze in strutture e servizi pubblici e privati accreditati, al fine di verificare l'efficacia dell'indirizzo metodologico e teorico-culturale seguito dall'istituto" (art. 8, D.M. 509/98).

L'indicazione che emerge dal decreto è dunque di una particolare attenzione alla verifica di efficacia anche se non viene chiarito se tale efficacia vada riferita all'indirizzo psicoterapeutico o piuttosto al modello formativo in cui l'indirizzo viene sviluppato e che dovrebbe poi riflettersi sull'efficacia terapeutica degli specializzandi.

In particolare la "messa alla prova" dell'indirizzo scientifico durante "documentate esperienze in strutture e servizi pubblici e privati accreditati" (art. 8, D.M. 509/98) risulta però controversa in quanto non è possibile verificare se tale compito venga effettivamente svolto, pur non mancando strumenti e metodologie adeguati al riguardo (Dazzi,

${ }^{1}$ http://www.miur.it/006MENU_C/0012Docum/0098Normat/1964Regola_cf2.htm 
Ricerca in Psicoterapia / Research in Psychotherapy 2010; 1(13): 6-22. http://www.researchinpsychotherapy.net

Lingiardi, \& Colli, 2006; Rønnestad \& Ladany, 2006; O'Donovan \& Dawe, 2002).

La cultura condivisa della verifica dell'efficacia delle terapie, ampiamente diffusa in ambito internazionale (Lambert, 2003; Lambert \& Ogles, 2004; Norcross, Beutler, \& Levant, 2005; Bennett-Levy, McManus, Westling \& Fennell, 2009), è poco presente in Italia, pur tra alcune lodevoli eccezioni (Dazzi, Lingiardi, \& Colli, 2006; Di Nuovo, Lo Verso, Blasi, \& Giannone, 2003). D'altra parte appare invece ancora inesplorata la valutazione dell'efficacia dei modelli formativi.

Parlando di formazione diventa inevitabile focalizzare l'attenzione sui protagonisti in gioco: da un lato le scuole e i docenti, terapeuti esperti, dall'altro i trainee, spesso giovani psicologi, più raramente medici.

L'esercizio della professione psicoterapeutica è diventata, infatti, sempre più un obiettivo per molti psicologi che, spesso subito al termine del percorso universitario, sentono la necessità di completare la propria professionalità.

D'altra parte questa scelta viene sentita da molti psicologi quasi come obbligata, data la saturazione del mercato del lavoro, e i dati sembrano confermare questa sensazione; l'Italia con i suoi 64142 psicologi (dato aggiornato al 31 dicembre 2007 ) ha ormai superato il rapporto di 1 psicologo ogni 1000 persone (Tonzar, 2007), dati presentati al convegno della commissione tecnico-consultiva per le scuole di psicoterapia del 22 marzo $2007^{2}$, e questo rapporto è destinato inesorabilmente a crescere progressivamente dato l'alto numero di iscritti alle facoltà di Psicologia (circa 70 000) (fonte Ordine Nazionale degli Psicologi, 2007).

A fronte di questa richiesta crescente di formazione sono nate numerosissime scuole che hanno inoltrato al MIUR e concluso positivamente l'iter di riconoscimento.

Attualmente il numero di scuole riconosciute dal Ministero dell'università è di 341 di cui 204 sedi principali e 137 sedi periferiche (dato aggiornato a giugno 2009); dal 2002 al 2009 l'incremento è stato di 124 scuole, pari al $57.1 \%$ (Tabella 1 ).

Tabella 1. Numero delle scuole e distribuzione geografica (slide tratta dalla presentazione del prof. Rubini, disponibile sul sito MIUR, integrata con i dati del 2009).

\begin{tabular}{lcccc} 
& Nord & Centro & Sud & Totale \\
\hline $\mathbf{2 0 0 2}$ & 74 & 92 & 51 & 217 \\
$\mathbf{2 0 0 7}$ & 104 & 128 & 73 & 305 \\
$\mathbf{2 0 0 9}$ & 113 & 146 & 82 & 341
\end{tabular}

${ }^{2}$ http://www.miur.it/0002Univer/0466Docume/6454Conveg cf2.htm 
Ricerca in Psicoterapia / Research in Psychotherapy 2010; 1(13): 6-22. http://www.researchinpsychotherapy.net

Osservando la distribuzione delle sedi principali sul territorio nazionale emerge una sostanziale omogeneità sul territorio, anche se andando a vedere più in dettaglio la localizzazione delle 341 scuole emerge come Roma e Milano siano le città che ospitano il maggior numero di scuole (circa 50), seguite da Napoli, Torino, Firenze, Padova e Bologna (tra le 10 e le 20).

A partire dal 2002 è stato fissato in 20 il numero massimo di allievi per ogni scuola; di fatto il numero di allievi oscilla tra 10 e 20 con una media di 18 che moltiplicato per il numero delle scuole si traduce in 6030 potenziali terapeuti in formazione ogni anno.

È un numero esagerato per un Paese come l'Italia che ha già un rapporto di $0.61-0.65$ psicoterapeuti per 1000 abitanti, tra $i$ più alti in Europa insieme ad Austria (0.69) e Germania (0.62) (Zerbetto \& Tamtam, 2001; Tonzar, Ordine Nazionale Psicologi, 2006). Inoltre considerando il numero di psicologi che si laureano ogni anno (6851 nel 2008, di cui 2744 con laurea specialistica; fonte: sito MIUR) emerge come esista una forte sproporzione tra richiesta e offerta.

Lasciando da parte i numeri va comunque evidenziato che quella di intraprendere una specializzazione in psicoterapia è una scelta importante che determina per i futuri trainee un impegno oneroso in termini di tempo e di denaro.

Tuttavia non sempre nel così grande numero di scuole presenti sul territorio è garantito un adeguato livello formativo né d'altra parte all'utente naïve che si avvicina per la prima volta alle scuole, vengono forniti strumenti di valutazione o indici che siano di aiuto per orientarsi in un così variegato panorama formativo. Per esempio, non esistono dati sul livello occupazionale degli psicoterapeuti, né sull'efficacia dei programmi formativi o più semplicemente sulla soddisfazione degli specializzandi, con rare eccezioni (Baietta et al., 2009, http://www.apc.it/).

Pertanto i futuri trainee si trovano nella situazione di dover scegliere la scuola di specializzazione raccogliendo informazioni relative all'orientamento teorico, ai programmi didattici, ai docenti, alle sedi e in particolare ai costi. Tra le modalità di ricerca di informazioni, un ruolo primario è rivestito da Internet, per la sua facilità di accesso e per la sempre maggiore familiarità che gli utenti hanno con questo strumento.

Tale risorsa rappresenta, infatti, un canale semplice e accessibile di raccolta di informazioni da parte di una popolazione piuttosto giovane, costituita generalmente da neolaureati, affiancando altre modalità come le richieste di informazioni fatte tramite e-mail o per telefono, gli incontri di presentazione o selezione fatti dalle scuole stesse, il passaparola con altri trainee già in formazione.

Tuttavia, se guardiamo le informazioni che le scuole forniscono sui loro siti, ci rendiamo conto che i colloqui di selezione sono in quasi un quarto dei casi a pagamento. $\grave{E}$ ragionevole pensare che nella maggioranza dei casi tali colloqui vengano presi in considerazione come 
Ricerca in Psicoterapia / Research in Psychotherapy 2010; 1(13): 6-22. http://www.researchinpsychotherapy.net

fonte di informazione solo quando ormai la scelta è stata fatta. Il web quindi sembrerebbe rappresentare un canale informativo privilegiato per i futuri trainee oltre che un importante spazio di visibilità per le scuole stesse.

$\mathrm{Su}$ Internet è possibile trovare differenti siti che mettono a disposizione un elenco delle scuole private di psicoterapia, tuttavia il più completo e aggiornato è, ovviamente, quello del Ministero dell'università e della ricerca (www.miur.it) che oltre a fornire un elenco in formato pdf di tutte le scuole e la normativa di riferimento che regola le scuole stesse, mette a disposizione un motore di ricerca che consente di individuare le scuole attive in ogni regione.

Altri siti che forniscono un elenco delle scuole, anche se non sempre aggiornato come quello del Ministero, sono quelli delle tre associazioni che raggruppano le scuole private: la Federazione italiana delle associazioni di psicoterapia (www.fiap.info), la Federazione delle associazioni italiane di psicoterapia (www.faipnet.it), il Coordinamento nazionale delle scuole private di psicoterapia (www.cnspscuolepsicoterapia.it) e lo stesso fanno anche alcuni siti di argomento psicologico ma non istituzionali come : www.psychomedia.it, www.psicologia-psicoterapia.it, $\quad$ www.scuoledipsicoterapia.it, www.scuoledipsicoterapia.opsonline.it, www.psiconline.it, In ogni caso non vengono fornite informazioni dirette sulle scuole ma solo indicazioni sulla sede, i riferimenti telefonici o Internet, pertanto gli studenti devono cercare autonomamente tra i siti delle singole scuole le informazioni necessarie a orientarsi.

L'obiettivo del lavoro è quello di valutare la qualità e la completezza delle informazioni relative alle scuole di specializzazione in psicoterapia disponibili su Internet, mettendosi nella prospettiva dei trainee, per evidenziare innanzitutto i punti di forza e le criticità. In secondo luogo, per mettere i futuri trainee nelle migliori condizioni di scelta sottolineando alcune criticità di cui tenere conto e infine per orientare in futuro le scuole stesse verso una maggiore attenzione alla visibilità e all'aggiornamento dei contenuti della scuola, anche attraverso la promozione di attività di ricerca nella scuola e sulla scuola.

\section{Metodologia}

Il lavoro si è focalizzato sull'analisi dei siti web di 201 scuole di specializzazione riconosciute dal MIUR (fino a gennaio 2009), valutando la presenza/assenza di alcuni dati relativi alle caratteristiche della scuola.

La raccolta delle informazioni è stata focalizzata sulle seguenti tematiche: indirizzo teorico; impegno orario (quando si svolgono le lezioni? Che durata hanno?); tirocinio (quante ore di tirocinio sono previste? In quali strutture?); analisi personale (è prevista dal piano di studi? Per quante ore? Quali sono i costi?); supervisioni (quante ore sono 
Ricerca in Psicoterapia / Research in Psychotherapy 2010; 1(13): 6-22. http://www.researchinpsychotherapy.net

previste? Individuale/di gruppo? Quali sono i costi?); regolamento (è indicato il regolamento della scuola?); bibliografia di riferimento (sono indicati gli autori e i testi di riferimento?); costi (quanto costa il corso? Cosa comprende? $\mathrm{Ci}$ sono costi aggiuntivi?).

Le aree indagate sono state categorizzate in variabili dicotomiche (caratteristica presente o assente) e dove possibile sono state quantificate in variabili continue (ore di tirocinio, di supervisione, di analisi personale, costi). Nella lettura dei dati è importante sottolineare che il quadro che emerge è relativo a ciò che viene riportato dai siti nelle scuole e non necessariamente alla reale situazione della scuola. Infatti, la presenza di una caratteristica sta a indicare che il sito della scuola la riporta esplicitamente, mentre l'assenza indica che la caratteristica in oggetto non viene indicata esplicitamente, ma non necessariamente che la scuola non la preveda (ad esempio dai dati emerge che 28 sedi principali prevedono colloqui di selezione a pagamento, questo significa che anche altre scuole potrebbero prevederlo ma sul sito questo non è indicato, lasciando intendere che è gratuito).

La rilevazione si è svolta a gennaio/febbraio 2009, pertanto i dati si intendono aggiornati a tale data.

\section{Risultati}

I risultati sono stati strutturati per chiarezza di esposizione in 3 aree tematiche: l'organizzazione generale delle scuole, la didattica e i costi. Le analisi presentate sono relative a distribuzioni di frequenza per ognuno degli indicatori descritti in precedenza e dove possibile sono state indicate le statistiche descrittive (per gli indicatori su variabili continue come i costi, il monte ore di tirocinio, di supervisione e di analisi personale). Il numero delle scuole di formazione in psicoterapia considerate nell'analisi è di 201 sedi principali (si tratta di scuole private riconosciute dal MIUR conformemente alla legge 509 del 1998) a cui si aggiungono 132 sedi periferiche (scuole attivate da alcune sedi centrali ma dislocate in regioni differenti che con le sedi centrali condividono il paradigma teorico e le modalità didattiche). Sono $68(33.3 \%)$ le sedi centrali che hanno chiesto e ottenuto nel corso degli anni l'attivazione di sedi periferiche, con un numero variabile tra 1 a 7 (Tabella 2).

Considerando la distribuzione delle 333 scuole rispetto all'orientamento teorico (fatta sulla base di quanto indicato sui siti delle scuole), quello maggiormente presente risulta essere quello psicoanalitico/psicodinamico, che rappresenta circa il 33\% delle scuole, seguito da quello da quello sistemico relazionale (circa il $21 \%$ ) e da quello cognitivo/cognitivo-comportamentale (circa il 19\%) e (Tabella 3). 
Ricerca in Psicoterapia / Research in Psychotherapy 2010; 1(13): 6-22. http://www. researchinpsychotherapy.net

Tabella 2. Numero delle sedi periferiche attivate da ogni sede centrale (estrapolazione dai dati ricavabili dal sito MIUR).

\begin{tabular}{ccc}
\hline $\begin{array}{c}\mathbf{N}^{\circ} \text { Sedi } \\
\text { periferiche }\end{array}$ & Sedi centrali & $\%$ \\
\hline 0 & 136 & 66,7 \\
1 & 33 & 16,2 \\
2 & 20 & 9,8 \\
3 & 6 & 2,9 \\
4 & 3 & 1,5 \\
5 & 1 & 0,5 \\
6 & 3 & 1,5 \\
7 & 2 & 1,0 \\
Totale & 204 & 100 \\
\hline
\end{tabular}

Tabella 3. Distribuzione delle scuole perorientamento teorico (dati percentuali).

\begin{tabular}{lc}
\hline & $\%$ \\
\hline Psicoanalitico & 27,3 \\
Sistemico relazionale & 21,0 \\
Cognitivo comportamentale & 12,3 \\
Cognitivo & 6,6 \\
Gestalt & 5,7 \\
Psicodinamico & 5,7 \\
Integrato & 3,9 \\
Umanistico & 3,6 \\
Altro* & 13,8 \\
\hline
\end{tabular}

\footnotetext{
*Note. Include orientamenti non direttamente riferibili ai principali modelli (ad esempio analisi bioenergetica, psicodramma, psicoterapia breve strategica, psicosintesi, somato psicoterapia, terapia gruppanalitica, metodologia transculturale, biosistemica, psicosomatica, terapia funzionale, terapia comparata, terapia fenomenologico-esistenziale, terapia bionomica).
}

\subsection{L'organizzazione}

Il primo dato che emerge dall'analisi è che l'89.1\% (179) delle sedi principali considerate ha un sito Internet attraverso cui fornire informazioni; al momento della rilevazione uno di questi siti era però in costruzione. La durata delle scuole è di 4 anni, conformemente alla normativa vigente, tuttavia in 13 casi $(6.5 \%)$ viene richiesta la frequenza di un corso/anno propedeutico (con un costo variabile tra i $120 €$ e i 3000 
$€$; media $1191 €)$ che porta, di fatto, la durata complessiva del corso a 5 anni; la metà di queste scuole che richiedono un anno propedeutico è di tipo sistemico-relazionale.

Tutte le scuole prevedono uno o più colloqui di ammissione o prove di selezione che nella maggior parte dei casi sono gratuiti, in 29 casi $(14.4 \%)$ sono invece a pagamento con un costo variabile tra i $35 €$ e i $500 €$ (costo medio $149 €)$ che solo in alcuni casi viene rimborsato in caso di non ammissione (Tabella 4).

Tabella 4. Presenza percentuale di ciascuno degli indici considerati nel sito delle scuole.

\begin{tabular}{|c|c|c|}
\hline & Si & No \\
\hline Colloquio di ammissione/selezione & $100 \%$ & $0 \%$ \\
\hline Regolamento & $22,4 \%$ & $77,6 \%$ \\
\hline Bibliografia & $* 29,4 \%$ & $70,6 \%$ \\
\hline Calendario delle attività & $* * 42,9 \%$ & $57,1 \%$ \\
\hline Sedi di tirocinio & $9,2 \%$ & $91,8 \%$ \\
\hline Insegnamenti & $* * * 83,6 \%$ & $16,7 \%$ \\
\hline Nominativi dei docenti & $68,7 \%$ & $31,3 \%$ \\
\hline Supervisione & $73,1 \%$ & $25,9 \%$ \\
\hline Terapia personale & $* * * * 55,3 \%$ & $44,7 \%$ \\
\hline Costo annuale di iscrizione & $52,2 \%$ & $47,8 \%$ \\
\hline Costo orario della terapia personale & $2,6 \%$ & $97,4 \%$ \\
\hline \multicolumn{3}{|c|}{$\begin{array}{l}\text { Note. } \\
* \text { non è stata considerata esaustiva la sola indicazione degli autori di } \\
\text { riferimento, senza una chiara bibliografia degli stessi autori; } \\
\text { ** non sono state considerate esaustive indicazioni come " } 2 \text { week end al mese", } \\
\text { "20 fine settimana all'anno", ma solo quelle in cui erano indicati chiaramente } \\
\text { giorni di svolgimento delle lezioni; } \\
{ }^{* *} \text { di cui } 14,4 \% \text { indicata in modo esaustivo con programma, docente, } \\
\text { bibliografia e } 69,2 \% \text { indicata con un generico elenco di materie; } \\
* * * \text { di cui } 49.3 \% \text { indicata come obbligatoria e } 6.0 \% \text { consigliata. }\end{array}$} \\
\hline
\end{tabular}

\subsection{La didattica}

Nell'analisi degli aspetti più strettamente didattici della formazione è stato utilizzato come quadro di riferimento il modello di formazione tripartita (Botermans, 1996; Rønnestad \& Ladany, 2006) che identifica tre macroaree: la didattica frontale, la supervisione e l'analisi personale, con l'aggiunta della pratica clinica.

Le informazioni sulla didattica vengono indicate chiaramente da 29 scuole (pari al 14.4\%), mentre 139 scuole (pari al 69.2\%) indicano solo un generico elenco di aree di studio o materie di insegnamento, spesso analogo a quello riportato nella normativa ministeriale, ma senza alcuna specifica.

Nel complesso sono 147 (73.1\%) le scuole che menzionano chiaramente la supervisione come una delle attività svolte nella scuola, anche senza specificarne la durata. Guardando più in dettaglio l'indicazione delle ore 
Ricerca in Psicoterapia / Research in Psychotherapy 2010; 1(13): 6-22. http://www.researchinpsychotherapy.net

di supervisione emerge una situazione estremamente disomogenea che oscilla tra le 40 ore della scuola al livello inferiore alle 690 di quella che ne propone di più (la media risulta di 190.73, d.s. 131.28).

Rispetto alle attività di tirocinio sono stati considerati il monte ore annuale e le sedi di svolgimento. L'indicazione delle ore di tirocinio da svolgere annualmente viene dato dal $74 \%$ delle scuole (pari a 145) e il numero medio di ore annuali è di 140 (si varia da 100 a 300 ore), mentre l'indicazione delle sedi convenzionate in cui è possibile svolgere quest'attività viene pubblicata sul sito solo da 18 scuole $(9.2 \%)$; negli altri casi vengono indicate generiche "strutture pubbliche o private convenzionate".

L'analisi personale, spesso considerata un aspetto chiave nella formazione in psicoterapia, viene considerata obbligatoria da 99 scuole $(49.3 \%)$ mentre 12 scuole $(6.0 \%)$ la propongono specificandone la non obbligatorietà ai fini del titolo ma semplicemente consigliandola e infine 66 scuole $(32.8 \%)$ non la indicano pur non chiarendo se sia o non sia parte del percorso formativo (Tabella 5).

Tabella 5: Indicazione della terapia personale nei differenti orientamenti.

\begin{tabular}{lccc}
\hline & Obbligatoria & Indicata & Non indicata \\
\hline Altro & 55,0 & 10,0 & 35,0 \\
Cognitivo & 42,9 & 0,0 & 57,1 \\
Cognitivo comportamentale & 15,4 & 3,8 & 80,8 \\
Gestalt & 81,8 & 0,0 & 18,2 \\
Integrato & 66,7 & 11,1 & 22,2 \\
Psicoanalitico & 76,8 & 8,9 & 14,3 \\
Psicodinamico & 75,0 & 6,3 & 18,8 \\
Sistemico relazionale & 8,7 & 8,7 & 82,6 \\
Transazionale & 100,0 & 0,0 & 0,0 \\
Umanistico & 100,0 & 0,0 & 0,0 \\
Totale & 55,9 & 6,8 & 37,3 \\
\hline
\end{tabular}

Le ore di terapia personale variano da un minimo di 20 fino a 600 ore durante i 4 anni di scuola, con una media di 197 ore; tuttavia bisogna dire che solo 56 scuole $(27.9 \%)$ indicano sul sito la durata della terapia personale mentre le altre, pur indicandone la presenza nel percorso formativo, non ne specificano la durata minima o massima.

Il dato relativo al costo orario della terapia personale (range di 45-80 $€$; media di $53.8 €)$ viene indicato solo da 5 scuole (2.6\%) mentre quello della supervisione individuale viene indicato solo da una scuola $(60 €)$; in ogni caso il costo dell'analisi personale è sempre escluso dal costo di iscrizione e costituisce quindi una voce di spesa separata rispetto alla quota annuale.

Un'ulteriore dimensione legata alla didattica è quella dei docenti impiegati nei corsi; a questo riguardo emerge come l'indicazione dei 
Ricerca in Psicoterapia / Research in Psychotherapy 2010; 1(13): 6-22. http://www.researchinpsychotherapy.net

docenti venga fatta da 138 scuole $(68.7 \%)$ e il numero medio di docenti per scuola è di 32 , con un range che va da 9 a 82 . Va però evidenziato che talvolta si ritrova l'indicazione di docenti esterni o visiting professor, nazionali o internazionali, che in passato hanno tenuto seminari ma non è chiaro se annualmente tengano un intervento o semplicemente vengono inseriti come esempi di collaborazioni importanti una tantum.

\subsection{I costi}

Nel calcolare i costi delle scuole di psicoterapia sono stati considerati solo i costi diretti, relativi al contributo annuale di iscrizione, mentre non sono stati considerati tutti i costi indiretti. L'indicazione del costo della scuola si ritrova solo nel $52.2 \%$ dei siti e varia tra $1190 €$ e $5430 €$ (costo medio $3542 €$ ); il costo comprende sempre tutta la didattica frontale ed esperienziale mentre per quanto riguarda le supervisioni, l'analisi personale o di gruppo, la situazione è più articolata in quanto alcune scuole li includono nel costo annuale mentre altre no. Inoltre 23 scuole $(11.4 \%)$ prevedono, oltre al costo annuale, un costo di iscrizione che può essere annuale o una tantum che varia tra $50 €$ e $600 €$ (costo medio 208 $€)$ che può includere eventuali abbonamenti a riviste di settore o l'iscrizione alle società scientifiche di riferimento della scuola.

Accanto al costo di iscrizione è invece spesso presente un contributo per l'ammissione all'esame finale che generalmente ammonta a poche centinaia di euro ma anche in questo caso non è semplice avere un quadro completo della situazione.

Facendo un confronto relativo ai costi tra le scuole di differenti orientamenti (Tabella 6) si osserva come le scuole con un costo maggiore siano anche quelle che richiedono poi un minor numero di ore di terapia personale (con l'eccezione delle scuole transazionali) che, come riportato in precedenza, sono sempre escluse dal costo.

Tabella 6. Costo annuale $(€)$, ore annuali di tirocinio e ore complessive di terapia personale e supervisione suddivisi per orientamento teorico.

\begin{tabular}{lcccc}
\hline & $\begin{array}{c}\text { Costo } \\
\text { annuale }\end{array}$ & $\begin{array}{c}\text { Terapia } \\
\text { personale }\end{array}$ & Tirocinio & Supervisione \\
& & & & \\
\hline Cognitivo & 4100 & 27 & 148 & 155 \\
Cognitivo & 3973 & 25 & 151 & 165 \\
comportamentale & & & & \\
Gestalt & 3081 & 235 & 113 & 218 \\
Integrato & 3492 & 137 & 150 & 152 \\
Psicoanalitico & 3224 & 321 & 139 & 233 \\
Psicodinamico & 3663 & 169 & 143 & 178 \\
Sistemico relazionale & 3718 &. & 137 & 160 \\
Transazionale & 3623 & 80 & 167 & 278 \\
Umanistico & 3517 & 121 & 165 &. \\
Altro & 3508 & 167 & 131 & 135 \\
\hline
\end{tabular}


Ricerca in Psicoterapia / Research in Psychotherapy 2010; 1(13): 6-22. http://www.researchinpsychotherapy.net

\section{Discussione}

Dai dati presentati emerge innanzitutto che le scuole di psicoterapia hanno generalmente una visibilità molto ampia sul web, come dimostra il fatto che oltre il $90 \%$ ha un sito Internet, tuttavia, andando a guardare più da vicino, l'immagine che appare agli studenti in cerca di informazioni risulta nitida in alcuni casi e per alcuni aspetti, ma decisamente più sfocata per altri, arrivando fino al limite dell'assoluta oscurità.

I siti risultano generalmente aggiornati soprattutto per quanto riguarda l'apertura dei colloqui di selezione del nuovo anno o, più raramente, per la segnalazione di incontri o iniziative della scuola; non è negli scopi del presente lavoro una valutazione della qualità tecnica dei siti, della navigabilità e intelligibilità delle informazioni, tuttavia l'impressione generale è che accanto a siti facilmente navigabili si trovino spesso altri siti qualitativamente piuttosto scadenti in cui a volte non è facile recuperare le informazioni relative alle scuole di psicoterapia.

La presenza in 13 scuole di un corso/anno propedeutico al percorso quadriennale di specializzazione, che porta, di fatto, a 5 anni la durata dell'iter formativo, riveste un interesse particolare in relazione al recente riordino delle scuole di specializzazione universitarie che ha portato a 5 anni la durata di questi corsi, aprendo una riflessione sulla possibilità di rendere omogenea la durata anche delle scuole private.

In riferimento ai contenuti le informazioni più critiche sono senza dubbio quelle relative ai costi dal momento che l'impegno economico da affrontare è oneroso e richiede quindi un'attenta valutazione da parte dei giovani trainee; d'altra parte il fatto che circa il $50 \%$ delle scuole non indichi questo dato sui siti rappresenta un grosso limite. Tuttavia anche nel caso delle scuole che indicano il costo annuale, il dato risulta fuorviante o quantomeno incompleto per gli studenti che cercano di orientarsi, dal momento che la terapia personale non è mai inclusa nel costo annuale e che talvolta non lo sono nemmeno le supervisioni, a cui si aggiungono a volte anche altri costi collaterali.

Per quanto riguarda i costi indiretti i tentativi di quantificazione sono ancora più difficoltosi perché estremamente variabili, tuttavia comprendono varie voci tra cui i libri e i manuali, i trasporti e talvolta l'alloggio (per gli studenti che arrivano da altre città o regioni rispetto alla sede della scuola), oltre all'assicurazione per il tirocinio che a volte è già inclusa nelle quote di iscrizione e altre no.

In altre situazioni viene richiesto agli studenti di partecipare obbligatoriamente ai seminari organizzati dalla scuola fino all'effettiva specializzazione; altrove viene richiesta un'ulteriore analisi personale di alcune centinaia di ore per praticare la psicoterapia in autonomia.

È pur vero che la durata della terapia personale viene indicata talvolta in termini generici, con un minimo di ore, tuttavia questo non rende conto della mancata indicazione del costo orario medio della 
terapia o di un range di costi che dovrebbero invece essere definibili fin dall'inizio dal momento che spesso rappresentano una parte notevole del costo complessivo (considerando realistico il dato medio di $53 €$ /ora, una terapia personale di circa 100 ore ha un costo superiore al costo di iscrizione annuale).

In merito all'analisi personale colpisce poi l'attenzione il fatto che in ambito cognitivista, contrariamente a quanto generalmente si creda, la terapia personale venga indicata come obbligatoria da oltre il $40 \%$ delle scuole.

L'informazione che gli studenti ottengono dai siti Internet lascia intendere che le uniche scuole che richiedono sempre la terapia personale sono quelle a orientamento umanistico, mentre le altre la prevedono solo in parte; tuttavia questo non corrisponde necessariamente alla realtà dei fatti, per esempio circa il $20-25 \%$ delle scuole psicoanalitiche/psicodinamiche sembra non prevedere terapia personale mentre generalmente questi approcci la considerano un aspetto fondamentale della formazione. In tema di analisi personale o di gruppo è emersa inoltre una certa difficoltà nel distinguerla da quello che viene definito con varie accezioni "lavoro di gruppo sui propri aspetti personali", "riflessione di gruppo".

Un dato singolare è relativo a una scuola in cui la terapia personale può essere fatta anche da specializzandi del terzo e quarto anno a quelli del primo biennio, a costi più contenuti.

Lo stesso limite si presenta con la supervisione che se non sempre è quantificabile in termini temporali dovrebbe invece esserlo in termini di costo orario; invece non è possibile sapere quante ore sono dedicate alla supervisione, che spesso viene accorpata al monte ore pratico clinico, né se si tratta di supervisione individuale o di gruppo. Se rispetto alla supervisione fatta nella scuola qualche informazione, seppur parziale, si trovi nei siti, nessuna informazione si ha invece riguardo alla supervisione fatta durante il tirocinio, nelle strutture esterne alla scuola, che pure rappresenta un importante spazio di pratica clinica in cui più spesso si incontrano terapeuti di differenti orientamenti. Anche in questo caso non è possibile, infatti, quantificare il tempo dedicato alla supervisione né avere un riscontro sulla qualità dell'esperienza di tirocinio mentre è un aspetto che meriterebbe di essere adeguatamente monitorato. In merito al tirocinio colpisce poi il confronto con le scuole di specialità pubbliche che prevedono un monte ore tirocinio annuale variabile tra le 390 e le 600 ore rispetto alle 140 delle scuole riconosciute.

Il tema del costo della formazione chiama in causa direttamente il tema della qualità della stessa che allo stato attuale viene "monitorata" solo attraverso il passaparola con altri trainee in formazione; con rare eccezioni nel mondo cognitivo (Baietta et al., 2009), le scuole non mettono, infatti, a disposizione sul proprio sito Internet strumenti di valutazione della scuola stessa (soddisfazione degli studenti, livello di impiego lavorativo al termine del corso, protocolli di valutazione 
Ricerca in Psicoterapia / Research in Psychotherapy 2010; 1(13): 6-22. http://www.researchinpsychotherapy.net

dell'efficacia degli interventi terapeutici dei trainee, etc.) né d'altra parte esistono altre modalità di valutazione esterne (Ashworth, 1999; Ryan, Cullivan, \& Quayle, 2005).

Un secondo tema caldo è quello della didattica delle scuole che probabilmente riveste un ruolo anche maggiore rispetto a quello dei costi poiché determina in concreto la professionalità che i trainee andranno a utilizzare in contesti pubblici o privati e ha quindi delle conseguenze dirette sull'utenza. Dai dati emerge un quadro solo in parte soddisfacente perché se è vero che circa i due terzi delle scuole indicano un programma didattico dettagliato (con l'indicazione degli insegnamenti, delle ore dedicate, talvolta dei docenti) va però sottolineato che non è possibile né discriminare tra insegnamenti dedicati alle differenti fasi del ciclo di vita (bambini, adolescenti, adulti, anziani, famiglie, etc.) o alle famiglie di disturbi (dipendenze, disturbi dell'umore, disturbi alimentari, disturbi di personalità, etc.) né capire quanto spazio viene dato alla didattica frontale e quanto a quella esperienziale, o capire in cosa consista la didattica esperienziale (simulazioni di colloqui, role play, lavoro sulla dimensione corporea, etc.).

Questo secondo aspetto in particolare andrebbe chiarito anche alla luce del valore aggiunto che la pratica clinica e la didattica esperienziale danno alla formazione come peraltro già evidenziato oltre un decennio fa da Norcross (1996).

Inoltre nella maggior parte dei casi non è possibile quantificare con esattezza il tempo dedicato alla supervisione né se si tratta di supervisione individuale o di gruppo.

Un'ultima considerazione riguarda la presenza in 57 scuole su 201 (pari al 28.4\%) di corsi di counselling, che si affiancano a quelli in psicoterapia. $\grave{E}$ un dato che differisce sostanzialmente da quello riportato da Di Fabio (2008) che ne individua 43 in un lavoro aggiornato a giugno 2007 e che senza dubbio invita a riflettere sul doppio binario di formazione che esiste in $1 / 3$ delle scuole che formano professionisti della salute mentale con professionalità che spesso sono erroneamente confuse o sovrapposte e la cui formazione viene spesso fatta dagli stessi docenti. Peraltro questo dato invita ad aprire una discussione seria sul rapporto tra psicoterapia e counselling anche in vista di prossimi sviluppi normativi.

\section{Conclusioni}

Il lavoro, fornendo uno sguardo ampio dell'organizzazione delle scuole di psicoterapia, ha evidenziato alcuni punti di forza e alcune criticità relative al sistema di formazione oltre che la grande articolazione che lo caratterizza.

In particolare affinché i siti internet delle scuole diventino uno strumento utile non solo a fini pubblicitari, ma anche uno spazio 
realmente informativo per i giovani trainee in cerca di orientamento, è auspicabile da un lato l'integrazione delle informazioni riportate sui siti internet in modo da renderle il più possibile chiare e complete, in particolare negli aspetti della didattica e in quelli economici, dall'altro la creazione di un database complessivo che fornisca in modo immediato, chiaro e aggiornato le informazioni più rilevanti sulla formazione in psicoterapia.

La messa a disposizione di una guida generale e di facile lettura metterebbe gli studenti nella condizione di potersi orientare pienamente in un panorama formativo decisamente complesso, favorendo in questo modo una scelta consapevole e informata della scuola, lasciando poi ai siti delle singole scuole le informazioni più specifiche relative alle base teoriche, agli insegnamenti, ai docenti e alle iniziative della scuola.

In secondo luogo è opportuno cominciare a pensare anche in termini di qualità della formazione, con iniziative sia dal basso (valutazione da parte dei trainee del percorso formativo, dei tirocini) che dall'alto, attraverso la messa a punto di progetti di valutazione del percorso formativo da parte delle singole scuole sia in termini di efficacia dei trainee nell'attività clinica, sia in merito all'acquisizione di competenze tecniche e relazionali più specifiche (la capacità di costruire e mantenere una relazione terapeutica, di riconoscere e intervenire sulle rotture dell'alleanza, di fare un assessment e di condurre un primo colloquio, di utilizzare tecniche di intervento proprie dell'orientamento della scuola, la conoscenza dei modelli di trattamento basati sull'evidenza, etc.).

Infine una spinta in questa direzione può venire dalle scuole stesse attraverso la promozione all'interno del percorso formativo dell'attività di ricerca con e sui trainee.

\section{Bibliografia}

Ashworth, P., Williams, C., \& Blackburn, I.M. (1999). What becomes of cognitive therapy trainees? A survey of trainees' opinions and current clinical practice after postgraduate cognitive therapy training. Behavioural and Cognitive Psychotherapy, 27, 267-277.

Baietta, F., Carreri, M., Di Pauli, D., Gianello, A., Lanfredi, M., Mion, S., Morini, L., Pelaia, L., Scolaro, C., Untermarzoner, E., \& Perdighe, C. (2009). Qual è il destino professionale dei diplomati in psicoterapia della scuola della Associazione di Psicologia Cognitiva e della Scuola di Psicoterapia Cognitiva srl? From http://www.apc.it, http//www.scuola-spc.it/.

Bennett-Levy, J., McManus, F., Westling, B.E., \& Fennell, M. (2009). Acquiring and refining CBT skills and competencies: which training methods are perceived to be most effective?. Behavioural and Cognitive Psychotherapy, 37, 571-583. 
Botermans, J.F. (1996). The training of psychotherapists: Impact of confidence and mastery, self-efficacy, and emotional reactivity. Unpublished doctoral dissertation, Université catholique de Louvain, Belgium.

Dazzi, N., Lingiardi, V., \& Colli, A. (Eds.). (2006). La ricerca in psicoterapia. Modelli e strumenti. Milano: Raffaello Cortina.

Di Fabio, A. (2008). La formazione nel counseling in Italia: riflessioni a margine di una ricerca empirica. Counseling, $1(1)$.

Di Nuovo, S., Lo Verso, G., Di Blasi, M., \& Giannone, F. (Eds.). (2003). Valutare le psicoterapie: la ricerca italiana. Milano: Franco Angeli.

Lambert, M.J., \& Ogles, B.M. (2004). The efficacy and effectiveness of psychotherapy. In M.J. Lambert (Ed.), Bergin and Garfield Handbook of Psychotherapy and Behavior Change (5th ed.) (pp. 139-193). New York: Wiley.

Lambert, M.J. (2003). Bergin and Garfield Handbook of Psychotherapy and Behaviour Change (5th ed.). New York: Wiley.

Norcross J.C., Beutler, L.E., \& Levant R.F. (Eds.). (2005). Evidencebased practices in mental health: Debate and dialogue on the fundamental questions. Washington, DC: American Psychological Association Press.

Norcross, J. (1996). Training of psychotherapists. Acta psychiatrica belgica, 96, 218-237.

O'Donovan, A., \& Dawe, S. (2002). Evaluating training effectiveness in psychotherapy: lessons for the AOD field. Drug and Alcohol Review, $21,239-245$.

Orlinsky, D.E., \& Rønnestad, M.H. (2005). How Psychotherapists Develop: A Study of Therapeutic Work and Professional Growth. Washington DC: American Psychological Association Press.

Orlinsky, D.E. (2008, June). Psychotherapeutic professions as contexts of therapeutic practice and research I: current situation of the professions in varied countries. Paper presented at 39th International meeting SPR, Barcelona, Spain

Ryan, D., Cullivan, V., \& Quayle, E. (2005). A survey of trainees' opinions and current clinical practice after behaviour therapy training. Journal of Psychiatric and Mental Health Nursing, 12, 199208.

Rønnestad, M.H., \& Ladany, N. (2006). The impact of psychotherapy training: Introduction to the special section. Psychotherapy Research, $16(3), 261-267$.

Zerbetto, R., \& Tamtam, D. (2001). The Survey of European Psychotherapy Training 3: what psychotherapy is available in Europe?. European Journal of Psychotherapy \& Counselling, 4(3), $397-405$. 


\section{Lights and shades in the psychotherapy training}

Abstract: Training in psychotherapy is today for many clinical psychologists an important and almost forced step to complete their professionalism; in the face of this growing demand many private training schools are born, recognized by the Italian Ministry of University (MIUR), which are flanked to the traditional public schools. To navigate in such a complex landscape, an instrument of access to schools of different orientations is represented by the web sites of each of them followed by telephonic information, e-mail and interviews for admission made by the schools. The question we are asking is whether the student-future users are able to make an informed choice, also because of the huge commitment of time and money that is required. The work has analyzed the websites of 201 schools of specialization recognized by the MIUR, considering the presence of certain data concerning the characteristics of the school. In general, the sites are sufficiently up to date, mainly in relation to screening interviews for the new year; however, some critical information (e.g. costs, personal therapy, supervision) are unclear, making often hard for the students the choice.

Key words: training; psychotherapy; quality schools; website. 